\title{
A Maximum Principle Result for a General Fourth Order Semilinear Elliptic Equation
}

\author{
A. Mareno \\ Penn State University, Middletown, USA \\ Email: aum24@psu.edu
}

Received 2 June 2016; accepted 27 August 2016; published 30 August 2016

Copyright (C) 2016 by author and Scientific Research Publishing Inc.

This work is licensed under the Creative Commons Attribution International License (CC BY).

http://creativecommons.org/licenses/by/4.0/

(c) (i)

Open Access

\begin{abstract}
We obtain maximum principles for solutions of some general fourth order elliptic equations by modifying an auxiliary function introduced by L.E. Payne. We give a brief application of these maximum principles by deducing apriori bounds on a certain quantity of interest.
\end{abstract}

Keywords

Nonlinear, Fourth Order, Partial Differential Equation, Semilinear

\section{Introduction}

In [1], Payne obtains maximum principle results for the semilinear fourth order elliptic equation

$$
\Delta^{2} u=f(u)
$$

by proving that certain functionals defined on the solution of (1) are subharmonic. In this work, functionals containing the terms $\left|\nabla^{2} u\right|-u_{, i} \Delta u_{, i}$ are utilized and apriori bounds on the integral of the square of the second gradient and on the square of the gradient of the solution are deduced. Since then, many authors [2]-[11] and references therein have used this technique to obtain maximum principle results for other fourth order elliptic differential equations whose principal part is the biharmonic operator.

Other works deal with the more general fourth order elliptic operator $L^{2} u$, where $L u:=a_{i j} u_{i j}$ and $a_{i j}=a_{j i}$. In [12], Dunninger mentions that functionals containing the term $(\mathrm{Lu})^{2}$ can be used to obtain maximum principle results for such linear equations as

$$
L^{2} u+a L u+b u=0 .
$$

A similar approach is taken in [13] for a class of nonlinear fourth order equations.

In this paper, we modify the results in [1] and a matrix result from [14] to deduce maximum principles defined on the solutions to semilinear fourth order elliptic equations of the form: 


$$
L^{2} u=f(u) .
$$

Then we briefly indicate how these maximum principles can be used to obtain apriori bounds on a certain quantity of interest.

\section{Results}

Throughout this paper, the summation convention on repeated indices is used; commas denote partial differentiation. Let $a_{i j}(x)$ be a symmetric matrix. Moreover let $L u:=a_{i j} u_{i j}$, be a uniformly elliptic operator, i.e, the symmetric matrix $a_{i j}(x)$ is positive definite and satisfies the uniform ellipticity condition:

$a_{i j}(x) v_{i} v_{j} \geq|v|^{2}, x \in \Omega, v \in R^{n}$, where $\Omega$ is a bounded domain in $R^{n}$ and $n \geq 2$.

Let $u$ be a $C^{5}$ solution to the equation

$$
L^{2} u=f(u) \text { in } \Omega .
$$

where $f$ is say, a $C^{1}$ function. Now we define the functional

$$
P=c_{1}(L u)^{2}-\left(a_{m n} L u\right)_{, n} u_{, m}+c_{2}|\nabla u|^{2}+2\left(1-c_{1}\right) \int_{0}^{u} f(s) \mathrm{d} s+\beta(x) .
$$

We show that $L(P)$ is subharmonic and note that the constants $c_{1}$ and $c_{2}$ and any constraints on $f$ are yet to be determined.

By a straight-forward calculation, we have

$$
\begin{aligned}
P_{, i}= & 2 c_{1} L u L u_{, i}-\left(a_{n m} L u\right)_{, n i} u_{, m}-\left(a_{m n} L u\right)_{, n} u_{, m i} \\
& +2 c_{2} u_{, m} u_{, m i}+2\left(1-c_{1}\right) f(u) u_{, i}+\beta_{, i} .
\end{aligned}
$$

Now we write

$$
\begin{aligned}
L(P)= & a_{i j} P_{, i j} \\
= & 2 c_{1} a_{i j} L u L u_{, i j}+2 c_{1} a_{i j} L u_{, i} L u_{, j}-a_{i j}\left(a_{m n} L u\right)_{, n i j} u_{, m} \\
& -a_{i j}\left(a_{m n} L u\right)_{, n i} u_{, m j}-a_{i j}\left(a_{n m} L u\right)_{, n j} u_{, m i}-a_{i j}\left(a_{m n} L u\right)_{, n} u_{, m i j} \\
& +2 c_{2} a_{i j} u_{, m i} u_{, m j}+2 c_{2} a_{i j} u_{, m} u_{, m i j}+2\left(1-c_{1}\right) f(u) a_{i j} u_{, i j} \\
& +2\left(1-c_{1}\right) f^{\prime}(u) a_{i j} u_{, i} u_{, j}+L(\beta) .
\end{aligned}
$$

By expanding out the derivative terms in parentheses, we see that $L(P)$ is

$$
\begin{aligned}
= & 2 c_{1} f(u) L u+2 c_{1} a_{i j} L u_{, i} L u_{, j}-a_{i j} u_{, m}\left[a_{m n, n i j} L u+a_{m n, n i} L u_{, j}+a_{m n, n j} L u_{, i}\right. \\
& \left.+a_{m n, n} L u_{, i j}+a_{m n, i j} L u_{, n}+a_{m n, i} L u_{, n j}+a_{m n, j} L u_{, n i}+a_{m n} L u_{, n i j}\right] \\
& -2 a_{i j} u_{, m j}\left(a_{m n, i} L u_{, n}+a_{m n} L u_{, n i}+a_{m n, n i} L u+a_{m n, n} L u_{, i}\right) \\
& -a_{i j} u_{, m i j}\left(a_{m n, n} L u+a_{m n} L u_{, n}\right)+2 c_{2} a_{i j} u_{, m i} u_{, m j}+2 c_{2} a_{i j} u_{, m} u_{, m i j} \\
& +2\left(1-c_{1}\right) f(u) L u+2\left(1-c_{1}\right) f^{\prime}(u) a_{i j} u_{, i} u_{, j}+L(\beta) .
\end{aligned}
$$

The terms in lines 2 and 3 above containing two or more derivatives of $L u$ can be rewritten using (3) in the form $A^{i j} f(u)=L u_{, i j}$, where $A^{i j}$ denotes the matrix which is the inverse of the positive definite matrix $\left(a_{i j}\right)$. Furthermore, we use the identity $a_{i j} u_{, m i j}=L u_{, m}-a_{i j, m} u_{, i j}$ to rewrite the last two terms in line 4. Hence,

$$
\begin{aligned}
L(P)= & 2 f(u) L u+2 c_{1} a_{i j} L u_{, i} L u_{, j}-f(u) u_{, m} a_{m n, n}-a_{i j} L u\left(u_{, m} a_{m n, n i j}+2 a_{m n, n i} u_{, m j}\right) \\
& -a_{m n, n} L u L u_{, m}+a_{m n, n} L u a_{i j, m} u_{, i j}-a_{m n} L u_{, n} L u_{, m}+a_{m n} a_{i j, m} u_{, i j} L u_{, n} \\
& -a_{i j} a_{m n} u_{, m}\left(A_{, j}^{n i} f(u)+A^{n i} f^{\prime}(u) u_{, j}\right)-2 a_{i j} a_{m n} u_{, m j} A^{n i} f(u) \\
& -2 a_{i j} a_{m n, i} u_{, m} A^{n j} f(u)-2 a_{i j} u_{, m} a_{m n, n i} L u_{, j}-2 a_{m n, n} a_{i j} u_{, m j} L u_{, i} \\
& -a_{i j} L u_{, n} u_{, m} a_{m n, i j}-2 a_{i j} L u_{, n} u_{, m j} a_{m n, i}+2 c_{2} a_{i j} u_{, m i} u_{, m j} \\
& +2 c_{2} a_{i j} u_{, m} u_{, m i j}+2\left(1-c_{1}\right) f^{\prime}(u) a_{i j} u_{, i} u_{, j}+L(\beta) .
\end{aligned}
$$


Using the identity above for $a_{i j} u_{\text {,mij }}$ and the additional identity, $a_{i j} A_{, j}^{n i}=-a_{i j, j} A^{n i}$, which can be obtained by computing $\left(a_{i j} A^{n i}\right)_{j}$, for the terms at the ends of lines 6 and 3 respectively, we obtain

$$
\begin{aligned}
L(P)= & L(\beta)+\left(1-2 c_{1}\right) a_{i j} f^{\prime}(u) u_{, i} u_{, j}-2 a_{m n, n} u_{, m} f(u)+2 c_{2} u_{, k} L u_{, k} \\
& +2 c_{2} a_{i j} u_{, i k} u_{, j k}-2 c_{2} a_{i j, k} u_{, i j} u_{, k}-a_{m n, n} L u L u_{, m}+a_{m n, n} L u a_{i j, m} u_{, i j} \\
& -a_{r s} a_{i j} u_{, m} a_{m n, n r s} u_{, i j}-2 a_{m n, n} a_{i j} L u_{, i} u_{, m j}-2 a_{i j} L u a_{m n, n i} u_{, m j} \\
& -2 a_{i j} a_{m n, i} L u_{, n} u_{, m j}+a_{m n} a_{i j, m} L u_{, n} u_{, i j}+\left(2 c_{1}-1\right) a_{i j} L u_{, i} L u_{j} \\
& -a_{i j} a_{m n, i j} u_{, m} L u_{, n}-2 a_{i j} a_{m n, n i} u_{, m} L u_{, j} .
\end{aligned}
$$

To show that $L(P)$ is nonnegative, we establish a series of inequalities based on the following one from [14]: Let $\left(s_{p k}\right)$ be any $n \times n$ matrix. From the inequality

$$
a_{i j}\left(u_{i k}+\frac{1}{2} A^{i p} s_{p k}\right)\left(u_{, j k}+\frac{1}{2} A^{j q} s_{q k}\right) \geq 0
$$

One can deduce

$$
a_{i j} u_{, k j} u_{, k i}+s_{k i} u_{, k i} \geq-\frac{1}{4} A^{p q} s_{p k} s_{q k} .
$$

Repeated use of (9) on terms in lines 2, 3, 4, 5 in (7) yields the following:

$$
\begin{gathered}
a_{i j} u_{, i k} u_{, j k}+a_{m n} a_{i j, m} L u_{, n} u_{, i j} \geq-\frac{1}{4} A^{p q}\left(a_{m n} a_{p i, m} L u_{, n} a_{r s} a_{q i, r} L u_{, s}\right) \\
a_{i j} u_{, i k} u_{, j k}-2 a_{i j} a_{m n, i} L u_{, n} u_{, m j} \geq-A^{p q}\left(a_{p m, r} a_{r i} L u_{, n} a_{q n, s} a_{s i} L u_{, m}\right) \\
a_{i j} u_{, i k} u_{, j k}-2 a_{i j} a_{m n, n i} L u u_{, m j} \geq-A^{p q}\left(L u a_{p s, s r} a_{r i} L u a_{q l, l w} a_{w i}\right) \\
a_{i j} u_{, i k} u_{, j k}+a_{m n, n} a_{i j, m} L u_{, n} u_{, i j} \geq-\frac{1}{4} A^{p q}\left(a_{m n, n} a_{p i, m} L u a_{r s, s} a_{q i, r} L u\right) \\
a_{i j} u_{, i k} u_{, j k}-a_{i j} a_{r s} a_{m n, n r s} u_{, i j} u_{, m} \geq-\frac{1}{4} A^{p q}\left(a_{r s} a_{p i} u_{, m} a_{m n, n r s} a_{l w} a_{q i} u_{, z} a_{z t, t l w}\right) \\
a_{i j} L u_{, i} L u_{, j}-2 a_{m n, n i} a_{i j} u_{, m} L u_{, j} \geq-A^{p q}\left(a_{s k} u_{, r} a_{r n, n s} a_{m q} u_{, p} a_{k w, w m}\right) \\
a_{i j} L u_{, i} L u_{j}-a_{i j} a_{m n, i j} u_{, m} L u_{, n} \geq-\frac{1}{4} A^{p q}\left(a_{i n} u_{, r} a_{r k, i n} a_{l m} u_{, q} a_{k p, l m}\right) \\
a_{i j} u_{, i k} u_{j k}-2 c_{2} a_{i j, m} u_{, m} u_{, i j} \geq-c_{2}^{2} A^{p q}\left(a_{p i, m} u_{, m} a_{q i, l} u_{, l}\right) \\
a_{i j} u_{, i k} u_{j k}-2 a_{i j} a_{m n, n} L u_{, i} u_{, m j} \geq-A^{p q}\left(a_{p r, r} a_{s i} L u_{, s} a_{q l, l} a_{m i} L u_{, m}\right)
\end{gathered}
$$

Furthermore, by completing the square, we obtain useful inequalities for the last two terms in line 1 and the third term in line 2 of (7):

$$
\begin{gathered}
2 c_{2} u_{, k} L u_{, k} \geq-c_{2} u_{, m} u_{, m}-c_{2} L u_{, m} L u_{, m} \\
-2 a_{m n, n} u_{, m} f(u) \geq-a_{r p, p} u_{, r} a_{s q, q} u_{, s}-f^{2} \\
-a_{m n, n} L u L u_{, m} \geq-a_{m p, p} L u a_{m q, q} L u-L u_{, m} L u_{, m}
\end{gathered}
$$

We add (10)-(21) and label the resulting inequality, for part of $L(P)$, as 


$$
\begin{aligned}
\hat{L}(P)= & 7 a_{i j} u_{i k} u_{j k}+2 a_{i j} L u_{, i} L u_{, j}+2 c_{2} u_{, k} L u_{, k}-2 a_{m n, n} u_{, m} f(u)+a_{m n} a_{i j, m} L i_{, n} u_{, i j} \\
& -2 a_{i j} a_{m n, i} L u_{, n} u_{, m j}-2 a_{i j} a_{m n, n i} L u u_{, m j}+a_{m n, n} a_{i j, m} L u_{, n} u_{, i j}-a_{i j} a_{r s} a_{m n, n r s} u_{, i j} u_{, m} \\
& -2 a_{m n, n i} a_{i j} u_{, m} L u_{, j}-2 c_{2} a_{i j, m} u_{, m} u_{, i j}-2 a_{i j} a_{m n, n} L u_{, i} u_{, m j}+a_{m n, n} L u a_{i j, m} u_{, i j} \\
\geq & -A^{p q}\left(a_{s k} u_{, r} a_{r n, n s} a_{m q} u_{, p} a_{k w, w m}\right)-\frac{1}{4} A^{p q}\left(a_{i n} u_{, r} a_{r k, i n} a_{l m} u_{, q} a_{k p, l m}\right) \\
& -c_{2}^{2} A^{p q}\left(a_{p i, m} u_{, m} a_{q i, l} u_{, l}\right)-A^{p q}\left(a_{p r, r} a_{s i} L u_{, s} a_{q l, l} a_{m i} L u_{, m}\right) \\
& -A^{p q}\left(a_{p m, r} a_{r i} L u_{, n} a_{q n, s} a_{s i} L u_{, m}\right)-c_{2} u_{, m} u_{, m}-c_{2} L u_{, m} L u_{, m} \\
& -a_{r p, p} u_{, r} a_{s q, q} u_{, s}-a_{m p, p} L u a_{m q, q} L u-L u_{, m} L u_{, m}-a_{m n, n} L u L u_{, m}-f^{2} \\
& -\frac{1}{4} A^{p q}\left(a_{m n} a_{p i, m} L u_{, n} a_{r s} a_{q i, r} L u_{, s}\right)-\frac{1}{4} A^{p q}\left(a_{m n, n} a_{p i, m} L u a_{r s, s} a_{q i, r} L u\right) \\
& -\frac{1}{4} A^{p q}\left(a_{r s} a_{p i} u_{, m} a_{m n, n r s} a_{l w} a_{q i} u_{, z} a_{z t, t l w}\right)-A^{p q}\left(L u a_{p s, s r} a_{r i} L u a_{q l, l w} a_{w i}\right) .
\end{aligned}
$$

Now,

$$
\begin{aligned}
L(P)= & \hat{L}(P)+L(\beta)+\left(1-2 c_{1}\right) a_{i j} f u_{, i} u_{, j}+\left(2 c_{2}-7\right) a_{i j} u_{i k} u_{, j k}+\left(2 c_{1}-3\right) a_{i j} L u_{, i} L u_{, j} \\
\geq & \left(\left(2 c_{2}-7\right) a_{i j}-a_{m p, p} a_{m q, q} a_{i k} a_{j k}-\frac{1}{4} A^{p q} a_{m n, n} a_{p i, m} a_{r s, s} a_{q i, r} a_{i k} a_{j k}-A^{p q} a_{p s, s r} a_{r i} a_{q l, l w} a_{w i} a_{i k} a_{j k}\right) u_{i k} u_{, j k} \\
& +\left(\left(2 c_{1}-3\right) a_{i j}-\left(c_{2}+1\right) \delta_{i j}-A^{p q} a_{p j, r} a_{r l} a_{q i, s} a_{s l}-A^{p q} a_{p r, r} a_{i s} a_{q l, l} a_{j s}-\frac{1}{4} A^{p q} a_{m i} a_{p l, m} a_{r j} a_{q l, r}\right) L u_{, i} L u_{, j} \\
& +\left(\left(1-2 c_{1}\right) f^{\prime} a_{i j}-c_{2} \delta_{i j}-A^{j q} a_{s k} a_{i n, n s} a_{m q} a_{k w, w m}-c_{2}^{2} A^{p q} a_{p m, l} a_{q m, j}-\frac{1}{4} A^{p j} a_{r n} a_{i k, r n} a_{l m} a_{k p, l m}\right. \\
& \left.-a_{i p, p} a_{j q, q}-\frac{1}{4} A^{p q} a_{r s} a_{p m} a_{i n, n r s} a_{l w} a_{q m} a_{j t, t l w}\right) u_{i, i} u_{, j}+L(\beta)-f^{2} .
\end{aligned}
$$

Since $a_{i j}(x)$ is positive definite, for a sufficiently large value of $c_{2}$, where $c_{2}$ depends on the coefficients $a_{i j}$ and their derivatives, and for a sufficiently large value of $c_{1}$, say $(>1)$, where $c_{1}$ depends on the constants $c_{2}, \gamma, a_{i j}$, and various derivatives of $a_{i j}, L(P)$ can be made nonnegative as desired. Thus we have the following result.

Theorem 1. Suppose that $u \in C^{5}(\Omega) \cap C^{3}(\bar{\Omega})$ is a solution of (2) and $f \in C^{1}(R)$. If $f^{2} \leq \gamma$, where $\gamma>0, f^{\prime}(u) \leq \alpha, \alpha<0, \beta(x)$ is a nonnegative function such that $L(\beta) \geq \gamma$ then there exists positive constants $c_{2}$ and $c_{1}$ sufficiently large $\left(c_{1}>1\right)$ such that $P$ cannot attain its maximum value in $\Omega$ unless it is a constant.

We note that the function $f(u)=-\left(u+u^{3}\right)$ satisfies the conditions stated in Theorem 1 for a solution that is bounded above.

\section{Bounds}

Here we give a brief application of Theorem 1 .

Suppose that

$$
u=\frac{\partial u}{\partial n}=0 \text { on } \partial \Omega
$$

By Theorem 1,

$$
P \leq \max _{\partial \Omega}\left(c_{1}(L u)^{2}+\beta(x)\right) .
$$

Using integration by parts on the first two terms of $P$ yields the identity

$$
\int_{\Omega} a^{n m} u_{, k m} a^{k p} u_{, p n}-\left(a^{m n} L u\right)_{, n} u_{, m} \mathrm{~d} x=2 \int_{\Omega}(L u)^{2} \mathrm{~d} x .
$$


Upon integrating both sides of the previous inequality we deduce

$$
\begin{aligned}
& 2 \int_{\Omega}(L u)^{2} \mathrm{~d} x+2\left(1-c_{1}\right) \int_{\Omega}\left(\int_{0}^{u} f(s) \mathrm{d} s\right) \mathrm{d} x \\
& \leq\left[\max _{\partial \Omega}\left(c_{1}(L u)^{2}+\beta(x)\right)\right] \operatorname{area}(\Omega) .
\end{aligned}
$$

\section{References}

[1] Payne, L.E. (1976) Some Remarks on Maximum Principles. Journal d'Analyse Mathematique, 30, 421-433. http://dx.doi.org/10.1007/BF02786729

[2] Danet, C.-P. (2014) Two Maximum Principles for a Nonlinear Fourth Order Equation from Thin Plate Theory. Electronic Journal of Qualitative Theory of Differential Equations, 31, 1-9.

[3] Danet, C.P. (2011) Uniqueness in Some Higher Elliptic Boundary Value Problems in n Dimension Domain. Electronic Journal of Qualitative Theory of Differential Equations, 54, 1-12.

[4] Dhaigude, D.B., Dhaigude, R.M. and Lomte, G.C. (2012) Maximum Principles for Fourth Order Uniformly Elliptic Equations with Applications. International Journal of Applied Mathematical Research, 3, 248-258.

[5] Goyal, S. and Goyal, V.B. (2011) On Some Fourth Order Elliptic Boundary Value Problems. Applied Mathematical Sciences, 36, 1781-1793.

[6] Lomte, G.C. and Dhaigude, R.M. (2013) Maximum Principles for Fourth Order Semilinear Elliptic Boundary Value Problems. Malaya Journal of Matematik, 1, 44-48.

[7] Lu, W.D. and Wang, J.H. (1981) Maximum Principles for Some Semilinear Elliptic Equations of Fourth Order, and Their Application. Sichuan Daxue Xuebao, 4, 33-45. (In Chinese)

[8] Mareno, A. (2008) Maximum Principles for a Fourth Order Equation from Thin Plate Theory. Journal of Mathematical Analysis and Applications, 343, 932-937. http://dx.doi.org/10.1016/j.jmaa.2008.02.004

[9] Mareno, A. (2010) Integral Bounds for von Karmans Equations. ZAMM, 90, 509-513. http://dx.doi.org/10.1002/zamm.200900325

[10] Mareno, A. (2011) Maximum Principles for Some Higher Order Semilinear Elliptic Equations. Glasgow Mathematical Journal, 53, 313-320. http://dx.doi.org/10.1017/S001708951000073X

[11] Zhang, H. and Zhang, W. (2002) Maximum Principles and Bounds in a Class of Fourth Order Uniformly Elliptic Equations. Journal of Physics A: Mathematical and General, 35, 9245-9250. http://dx.doi.org/10.1088/0305-4470/35/43/318

[12] Dunninger, D.R. (1972) Maximum Principles for Solutions of Some Fourth Order Elliptic Equations. Journal of Mathematical Analysis and Applications, 37, 655-658. http://dx.doi.org/10.1016/0022-247X(72)90248-X

[13] Nichols, E.C. and Schaefer, P.W. (1987) A Maximum Principle In Nonlinear Fourth Order Elliptic Equations. Lecture Notes in Pure and Applied Mathematics, 109, 375-379.

[14] Schaefer, P.W. (1987) Pointwise Estimates in a Class of Fourth-Order Nonlinear Elliptic Equations. ZAMP, 38, 477479. http://dx.doi.org/10.1007/BF00944964

\section{Submit or recommend next manuscript to SCIRP and we will provide best service for you:}

Accepting pre-submission inquiries through Email, Facebook, LinkedIn, Twitter, etc.

A wide selection of journals (inclusive of 9 subjects, more than 200 journals)

Providing 24-hour high-quality service

User-friendly online submission system

Fair and swift peer-review system

Efficient typesetting and proofreading procedure

Display of the result of downloads and visits, as well as the number of cited articles

Maximum dissemination of your research work

Submit your manuscript at: http://papersubmission.scirp.org/ 